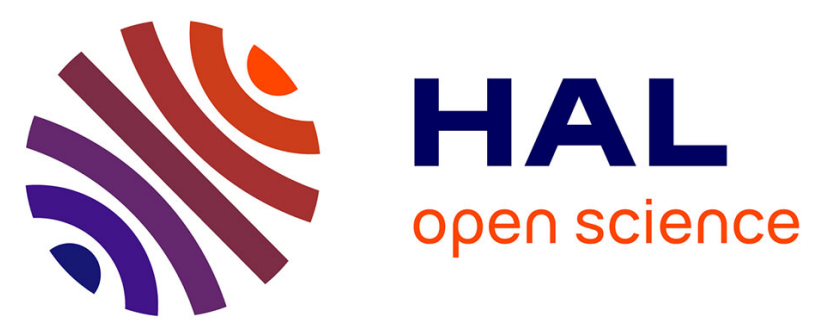

\title{
Reliability of parylene-based multi-electrode arrays chronically implanted on adult rat brains, and evidence of electrical stimulation on contact impedance
}

Napoleon Torres, David Ratel, Céline Cretallaz, Christophe Gaude, Sandrine Maubert, Jean-Louis Divoux, Christine Henry, David Guiraud, Fabien Sauter-Starace

\section{To cite this version:}

Napoleon Torres, David Ratel, Céline Cretallaz, Christophe Gaude, Sandrine Maubert, et al.. Reliability of parylene-based multi-electrode arrays chronically implanted on adult rat brains, and evidence of electrical stimulation on contact impedance. Journal of Neural Engineering, 2019, 10.1088/17412552/ab3836 . lirmm-02272772

\section{HAL Id: lirmm-02272772 \\ https://hal-lirmm.ccsd.cnrs.fr/lirmm-02272772}

Submitted on 28 Aug 2019

HAL is a multi-disciplinary open access archive for the deposit and dissemination of scientific research documents, whether they are published or not. The documents may come from teaching and research institutions in France or abroad, or from public or private research centers.
L'archive ouverte pluridisciplinaire HAL, est destinée au dépôt et à la diffusion de documents scientifiques de niveau recherche, publiés ou non, émanant des établissements d'enseignement et de recherche français ou étrangers, des laboratoires publics ou privés.

\section{(1) (1) $\$$}

Distributed under a Creative Commons Attribution - NonCommercial - NoDerivatives $\mid 4.0$ 
ACCEPTED MANUSCRIPT

\section{Reliability of parylene-based multi-electrode arrays chronically implanted on adult rat brains, and evidence of electrical stimulation on contact impedance}

To cite this article before publication: Napoleon Torres et al $2019 \mathrm{~J}$. Neural Eng. in press https://doi.org/10.1088/1741-2552/ab3836

\section{Manuscript version: Accepted Manuscript}

Accepted Manuscript is "the version of the article accepted for publication including all changes made as a result of the peer review process, and which may also include the addition to the article by IOP Publishing of a header, an article ID, a cover sheet and/or an 'Accepted

Manuscript' watermark, but excluding any other editing, typesetting or other changes made by IOP Publishing and/or its licensors"

This Accepted Manuscript is () 2019 IOP Publishing Ltd.

During the embargo period (the 12 month period from the publication of the Version of Record of this article), the Accepted Manuscript is fully protected by copyright and cannot be reused or reposted elsewhere.

As the Version of Record of this article is going to be / has been published on a subscription basis, this Accepted Manuscript is available for reuse under a CC BY-NC-ND 3.0 licence after the 12 month embargo period.

After the embargo period, everyone is permitted to use copy and redistribute this article for non-commercial purposes only, provided that they adhere to all the terms of the licence https://creativecommons.org/licences/by-nc-nd/3.0

Although reasonable endeavours have been taken to obtain all necessary permissions from third parties to include their copyrighted content within this article, their full citation and copyright line may not be present in this Accepted Manuscript version. Before using any content from this article, please refer to the Version of Record on IOPscience once published for full citation and copyright details, as permissions will likely be required. All third party content is fully copyright protected, unless specifically stated otherwise in the figure caption in the Version of Record.

View the article online for updates and enhancements. 


\title{
Reliability of parylene-based multi-electrode arrays chronically implanted on adult rat brains, and evidence of electrical stimulation on contact impedance
}

\author{
Authors
}

Napoleon Torres-Martinez ${ }^{1}$, David Ratel ${ }^{1}$, Celine Crétallaz ${ }^{1}$, Christophe Gaude ${ }^{1}$, Sandrine Maubert ${ }^{1+}$, Jean-Louis Divoux ${ }^{3}$, Christine Henry ${ }^{4}$, David Guiraud ${ }^{2}$, Fabien Sauter-Starace ${ }^{1}$

${ }^{1}$ Univ. Grenoble Alpes, CEA, Leti, CLINATEC, F-38000 Grenoble

${ }^{1+}$ Univ. Grenoble Alpes, CEA, Leti, DTBS, F-38000 Grenoble

2INRIA, University of Montpellier, CNRS, Montpellier, France

${ }^{3}$ Axonic, Sophia Antipolis, France

${ }^{4}$ SORIN CRM SAS (a Microport CRM company), Clamart, France

E-mail: fabien.sauter@cea.fr

Abstract

Objective: The goal of this study was to evaluate the long-term behavior of the surface electrode through electrochemical characterization and follow up of implanted parylene/platinum microelectrodes. Approach: To this aim, we designed and manufactured specific planar electrodes for cortical implantation for a rat model. This work was included in the INTENSE® project, one of the goals of which was to prove the feasibility of selective neural recording or stimulation with cuff electrodes around the vagus nerve. Main results: After a 12-week implantation on a rat model, we can report that these microelectrodes have withstood in-vivo, use. Regarding the biocompatibility of the electrodes (materials and manufacturing process), no adverse effect was reported. Indeed, after the three months implantation, we characterized limited tissue reaction beneath the electrodes and showed an increase and a stabilization of their impedance. Interestingly, the follow up of the electrochemical impedance combined with electrical stimulation highlighted a drop of the impedance up to $60 \% @ 1 \mathrm{kHz}$ after ten minutes of electrical stimulation at $110 \mathrm{~Hz}$.

Keywords: thin film, microelectrodes, impedance spectroscopy, chronic implantation, electrical stimulation, brain tissue response, biocompatibility

\section{Introduction}

One of the challenge of the neural interfaces is about the selective stimulation/recordings features. Indeed, the targeted neural tissues, either brain or nerves, are composed of underlying mid-scale structures such as functional and limited cortical areas and nerve fascicles. In both cases, the need for multi-contact ECoG which is an excellent compromise between invasiveness and signal quality for clinical use [1] or cuff electrodes gained growing interest. Our study was motivated by the focus on two topics: selective vagus nerve stimulation and advanced brain interfaces for both stimulation and recording. 
Vagus Nerve Stimulation (VNS) still motivates a growing interest due to the major possibility of providing therapies. VNS was initially designed for epilepsy management, and it has developed to encompass several other applications among which appetite regulation and heart failure [2][3][4]. In this particular application of VNS, one had to deal with side effects. Thus, either selective stimulation or closed-loop control based on nerve recordings can drastically increase the benefit over side effect ratio [5][6]. To achieve this goal, new cuff microelectrodes are needed, capable of selectively modulating specific fibers and extracting relevant information from selected axons. In the INTENSE project, in agreement with Plachta et al [7], we could discriminate compound action potentials with segmented cuff electrodes during acute experiments on mini-pigs [8].

To ensure an accurate geometry of the contact when resolution is taken to the micrometric scale, the use of processes derived from microelectronic is mandatory and silicone rubber is replaced with thin films of polyimide or parylene. The design engineer can mix several geometries on a single wafer, and significantly decrease the component price upon increasing the density of arrays on the wafer. However, there are new technical issues related to thin film materials: high impedance of the tracks, internal stresses and adhesion of the metal tracks.

When we started the INTENSE project, there were no FDA approved devices using polyimide in direct contact with the body, even though numerous studies [9][10][11][12] had already stated the potential of polyimide for the manufacture of neural interfaces. Parylene $\mathrm{C}$ was a potential substitute of polyimide, since published results on its compliance to the ISO10993-6 standard existed [13]. The feasibility of neuroprobes based on parylene $C$ has been reported with solid or mesh substrate [14], with a microfluidic channel [15] or with a bioresorbable stiffener [16]. However, their thermal budgets are limited compared to polyimide and the problem of delamination has to be tackled [17].

By accelerated ageing at $67^{\circ} \mathrm{C}$ in aqueous solution (PBS $1 \mathrm{X}$ ), the lifetime of electrodes manufactured by CEA Leti was estimated to be four and a half years [18]. In this paper, we investigate the long-term biocompatibility and the electrochemical impedance stability of the platinum/parylene electrode over time. To this end, we designed and tested multi-contact electrode arrays, and evaluated their level of biocompatibility and resistance to electrical stimulation using normal rats. Considering the risk of infection and failure of cuff electrodes with tibial or sciatic nerve connectors during long-term experiments, we decided to secure the study by choosing a cortical implantation. We also reduced mechanical stress and fatigue, or tissue reactions related to nerve geometry and motion using a flat array. Since the 2000s, scientists have tried to improve the understanding of the complex relationship between the invasiveness of electrodes (either surface or intracortical [19]) and electrical field effects at different currents [20], charge densities and frequencies [21]. Based on the characterization of electrodes and tissues after explantation, we gained preliminary consistent results about the reliability of parylene/platinum microelectrode stimulation. Finally, we bring original and complementary experimental data to the question related to the influence of an electrical stimulation on impedance which may compensate for the glial scar on a chronic implantation schema [22].

\section{Materials and methods}

\section{Electrode manufacture}

CEA Leti designed and manufactured 2-contact strips using MEMS techniques (deposition, photolithography and dry etching on a 200mm silicon carrier) in its ISO5 clean rooms, to minimize contamination. We added Atomic Layer Deposition (ALD) of titanium oxide and alumina to the process, to improve the long-term insulation of metal tracks. Contacts consisted of 6-rectangles of equal surface, which total surface is about $1 \mathrm{~mm}^{2}$ (Figure 1).

To reduce mechanical stress when stretching or folding the electrodes for peripheral nerve stimulation, we designed the electrical tracks in "serpentine". After release from the silicon substrate, to ease the 
surgical manipulation and to reduce the risk of failure due to connection/disconnection, we fixed the strips on a polyamide stand. We used Selective Laser Sintering from a biocompatible PA2200 powder (Initial ${ }^{\circ}$, Seynod, France) to 3D print this part, which comprised two rectangular extrusions so that the platinum contacts touch the duramater (so-called skates, see Figure 2 and Figure 3) and a superior platform to hold the connector (see Figure 2). We glued the two strips under the skates using a medical grade UV-sensitive acrylic glue (Vitralit 5140, Panacol®, Germany). Then, using Multi-Stranded PFACoated Stainless Steel Wire from A-M systems ${ }^{\circledR}$, Sequim (WA, USA), we wired the strips to an NPD $20 \mathrm{VV}$ connector (Omnetics®, Minneapolis, USA) using silver filled epoxy (Epo-tek H20E, Epoxy Technology, Billerica, USA).

\section{Cleaning and Sterilization}

After the clean room assembly process, we cleaned MEAs in an alcohol solution (70\%). Sterilization was carried out by exposure to ethylene oxide $\left(240\right.$ minutes, $\left.45^{\circ} \mathrm{C}, 850 \mathrm{mbar}\right)$, followed by a 3-day aeration process in a desorption chamber.

\section{Design of the experiment}

We selected twenty adult OFA rats (350g) (Charles River $\left.{ }^{\circledR}\right)$ for this 3-month experiment. We divided the group of rats into 4. Group A $(n=7)$ received the $E C o G$ contact strips in the epidural space of each hemisphere. Sham group B $(n=7)$ only received the stand, without the strips. Positive control group C $(\mathrm{n}=2)$ was implanted with RM-B (an abrasive material that produces reactive astrogliosis that serves for histological comparison ("RM-B" Polyurethane film containing $0.25 \%$ v/v zinc dibuthyldithiocarbamate, Hatano®, Japan)). Group D $(n=4)$ served as a negative control (not implanted) (Figure 3).

We performed electrochemical impedance spectroscopy (EIS) and electrical stimulation on group A $(\mathrm{n}=7)$; weekly for the six first weeks (starting 7 days post-implantation) and then every other week until week 12 (end of the experiment). We measured impedance between the working electrode in the contact and a counter electrode (cranial screws). Animals were anesthetized for each recording session, which comprised: (a) pre-stimulation electrode impedance spectroscopy, (b) electrical stimulation for 10 minutes, (c) post-stimulation electrode impedance spectroscopy. At week 9, we started investigating the recovery duration of the impedance and at the end of the 12-week protocol on rat \#9, we tested additionally a short stimulation (15-second long) to see if such a short pulse train could induce an impedance drop.

We performed all surgical procedures in agreement with the EU directive (2010/63/EU) on the care and use of laboratory animals and used procedures approved by the local committee (Cometh Grenoble number 266_Clinatec_NTM_10, Apafis\#709 N 2015052215592524.V4). Finally, we explanted the electrodes and then performed macroscopic observations on animal brains and characterized them by Scanning Electron Microscopy.

\section{Surgery procedure}

The animals were positioned separately in an induction chamber, and anesthesia was induced with $4 \%$ isoflurane (Isoflo 100\%, Axience SAS) in 100\% oxygen. Anesthesia was then maintained using 2\%, isoflurane by means of a facemask. We placed animals in a stereotaxic frame and a midline skin incision was made to expose the top of the skull. We performed rectangular bilateral craniotomies $(8.5 \mathrm{~mm} \mathrm{x}$ $3.5 \mathrm{~mm}$ ) in order to accommodate the contact array in the epidural space. We placed the systems (electrodes glued to the skates and wired to an Omnetics ${ }^{\circledR}$ connector) epidurally and centered them above the primary motor cortex. We placed three titanium screws in the frontal and parietal bones for fixation and these were used as a reference. We fixed this system to the skull bone with dental cement (Methax, Generique International ${ }^{\circledR}$, and France), see Figure 3. Pain was managed with subcutaneous injection of buprenorphine (Vetergesic ${ }^{\circledR} ;$ Sogeval, France) at a dose of $100 \mu \mathrm{g} / \mathrm{kg}$ and body temperature was monitored continuously. At the end of the procedure, we performed impedance spectroscopy on both hemispheres to compare contacts of the electrode arrays. 


\section{Electrochemical Impedance Spectroscopy}

We assessed the electrochemical behavior of the electrodes by means of Potentio Electrochemical Impedance Spectroscopy (PEIS) prior to implantation at room temperature in PBS 1X (solution diluted from PBS 10X, Sigma Aldrich, France reference P5493). We performed measurements using a SP200 Potentiostat (Bio-Logic $\odot$, Seyssinet-Pariset, France) with a two-electrode set-up: the reference and the counter electrode were both connected to the same platinum wire $(0.5 \mathrm{~mm}$ in diameter $)$ immersed in PBS $1 \mathrm{X}$. We applied a $50 \mathrm{mV}$ sinusoidal voltage with frequency swept from $1 \mathrm{~Hz}$ to $1 \mathrm{MHz}$ ( 6 points between each power of ten). Low frequency behavior was not investigated to avoid irreversible electrochemical reactions. We used Zfit module of EC-lab from Bio-Logic $\odot$ to identify the parameters of the equivalent model.

\section{Electrical stimulation features}

A dedicated hardware and developed by Sorin CRM®, Axonic ${ }^{\circledR}$ and INRIA, based on previous principle described in [23] [24] within the framework of the INTENSE project generated a stimulation pattern@110 Hz. Concerning this stimulation pattern, we chose a biphasic charge balanced pattern to limit potential tissue damage applied between E1 and E2 (Figure 3). Then, to avoid corrosion and to have the best chance to initiate action potentials, we introduced a $100 \mu \mathrm{s}$ interphase delay and applied the reversal current for longer than the stimulation current[20].

As sputtered platinum has a limited charge injection, prior to implantation we measured a maximum injectable charge of these electrodes in PBS at $20.2 \mu \mathrm{C} / \mathrm{cm}^{2}$ for $0.3 \mathrm{~ms}$ or $62.8 \mu \mathrm{C} / \mathrm{cm}^{2}$ for $1 \mathrm{~ms}$. The stimulation (Figure 4) is charge balanced, with an anodic pulse $(265 \mu \mathrm{A}, 1.36 \mathrm{~ms})$ followed by two cathodic pulses $(750 \mu \mathrm{A}, 0.24 \mathrm{~ms})$. Consequently, we had a charge density of $36 \mu \mathrm{C} / \mathrm{cm}^{2}$ per phase using $1 \mathrm{~mm} 2$ contacts which is below the lowest safety limit of platinum $\left(40 \mu \mathrm{C} / \mathrm{cm}^{2}\right.$ with passively balanced charge injection [25]) or [26] indicating [50-150 $\left.\mu \mathrm{C} / \mathrm{cm}^{2}\right]$ windows referring to [27] and [28].

\section{Data Analysis}

The impedance spectra analysis for each animal of group $A(n=7)$ was divided into non-stimulated contacts and stimulated contacts. Each animal had 2-contact strips on either side of the craniotomy. Electrodes E1 and E2 implanted in the left hemisphere underwent stimulation as explained above, while electrodes E3 and E4 were not stimulated. We defined the average of the measurements for nonstimulated contacts as the control to study the changes over time between the baseline impedance ( 1 week after surgery) and 12 weeks post-implantation. We performed 2-way Repeated Measured ANOVA (RMANOVA) tests to check the significance of the implantation time over impedance. For the analysis of stimulated contacts, we averaged pre-stimulation measurements and compared them with the poststimulation values, again using 2-way RMANOVA, follow by a post hoc (Bonferroni-Dunn) multiple comparison test. We performed this analysis during the first week and at the end of the experiment (week 12) to see if the implantation time has any impact on the acute stimulation effect on impedance. Typical impedance magnitudes ranged between $5 \mathrm{kOhm}$ and $20 \mathrm{kOhm}$ at $1 \mathrm{kHz}$ in-vivo. Thus we decided that data from contacts for which impedance magnitudes were above $200 \mathrm{kOhm}$ at $1 \mathrm{kHz}$ as non-informative and discarded them from the analysis.

\section{Scanning Electron microscopy (SEM)}

To image the electrode arrays and potential biological compounds after implantation, we used an $\mathrm{H}$ 4100 Hitachi (Tokyo, Japan) system. After explantation, we rinsed a batch of samples in PBS and dehydrated them stepwise with ethanol (10 minutes with concentrations of $70^{\circ}, 80^{\circ}, 90^{\circ}$ and then 20 minutes for $96^{\circ}$ ). Then, we dipped them in HMDS (Sigma-Aldrich, product reference 440191) for 5 minutes and dried them under aspiration for 3 minutes.

Long-term biocompatibility evaluation

\section{Euthanasia and tissue preparation}

Three months after implantation, we euthanized rats with isoflurane and, transcardially, with pentobarbital (Dolethal®, VÉTOQUINOL, France). We removed fixed rat brains with 
ParaFormaldehyde (Formaline 10\%, Sigma-Aldrich, product reference HT501128) and placed them in PBS $1 X$ with $30 \%$ added sucrose.

Histological analysis and immunohistochemistry

The procedure used has already been described [29]. In brief, after freezing, we cut brains serially into coronal sections using a freezing microtome (Leica Microsystem $\left.{ }^{\circledR}\right)$. Sections were collected and processed for Nissl staining and for immune histochemistry analysis for Glial Fibrillary Acidic Protein (GFAP), and Ionized calcium Binding Adapter molecule 1 (Iba1) for macrophage/microglia assessment. We incubated brain sections with the following primary antibody solutions overnight at $4{ }^{\circ} \mathrm{C}$ : anti-GFAP (DAKOcytomation, Glostrup, Denmark) to identify astrocytes and anti-Iba1 (Wako Chemicals GmbH®, Neuss, Germany). We diluted secondary antibodies (Molecular Probes - Alexa 488) directed against anti-GFAP and Iba1 and counter-stained all sections by incubation with the nuclear dye Propidium Iodide (Sigma Aldrich). We used sections treated with secondary antibody but not with primary antibody to determine non-specific binding. We mounted tissue sections with Fluorsave reagent (Merck Millipore ${ }^{\circledR}$, France) and visualized bound primary antibodies on a randomly defined set of slices using a confocal microscope. We carefully removed the samples from the bone and fixed them for analysis by scanning electron microscopy (SEM).

\section{Results}

In-Vitro EIS characterization in PBS

We performed electrochemical impedance spectroscopy in PBS $1 \mathrm{X}$ to control the consistency of contact behavior in a solution representing the extracellular medium. As expected, the behavior of the contact is resistive at high frequency (phase close to zero degree) and reached a minimal value of about minus 70 degrees at around $200 \mathrm{~Hz}$ associated to a capacitive behavior (Figure 5).

To simulate the electrochemical behavior of the platinum contact in PBS, we used a simple equivalent model with two resistances and a Constant Phase Element (CPE): $Z(f)=R_{1}+\frac{R_{2}}{R_{2} Q_{2}(j 2 \pi f)^{\alpha}+1}$ see schematic in Figure 5.

This model fits with the behavior of the contact in PBS (Figure 5). $\mathrm{R}^{2}$ values are 0.997 and 0.999 respectively for array number BR38 and BR43. Using the ZFit module from EC-Lab, $\alpha$, the exponent of the CPE, is identified at 0.8 (Table 1 ) meaning that the platinum system in PBS is strongly capacitive.

In-Vivo Results

We assessed systematically the impedance of twenty-eight epidural contacts in seven animals as described above (see methods). Error bars correspond to the mean standard error for each group $(n=7)$.

Non-stimulated contacts

To evaluate tissue evolution around the electrode due exclusively to the presence of the latter, we repeated Potentio Electrochemical Impedance Spectroscopy (PEIS) to the non-stimulated electrodes side over twelve weeks as described above. The results obtained indicated consistent changes in the electrode impedance spectrum as a function of time after implantation. The comparison between the mean baseline EIS and that 12 weeks post-implantation showed a significant variation in the impedance magnitude at $36.3 \mathrm{kHz}\left({ }^{*} \mathrm{p}<0.05\right)$ (Figure $\left.6 \mathrm{~A}\right)$. On the Bode diagram, the phase impedance progressively changed, with the arrival of a double hump (Figure 6B). Based on our data, we reported a maximum impedance increase in all animals at $1 \mathrm{kHz}$ after 2 weeks of implantation, followed by a partial decrease to reach a plateau finally (Figure 6C).

\section{Stimulated Contact}

At each session, in the left hemisphere of animals of group A, we applied 10 minutes of biphasic electrical stimulation on the epidural contacts. Here after, we sum up the general trends for EIS before and after stimulation: 
- Based on Figure 7C, we did not record significant change of the impedance of the stimulated electrodes over time. This result contrasts with the non-stimulated electrodes behavior (see Figure 6). However, impedance phase changed significantly between $200 \mathrm{~Hz}$ and $2,4 \mathrm{kHz}$ $(* \mathrm{*}<0,05)$;

- Based on Figure 8, we reported a significant $\left({ }^{*} p<0.05\right)$ impedance drop and phase evolution observed on the $[346 ; 512] \mathrm{Hz}$ frequency band at week 1 . At the end of the 12-week observation period, the impedance after stimulation still dropped significantly but on a wider frequency band [200; $2.4 \mathrm{k}] \mathrm{Hz}(* \mathrm{p}<0.05)$;

- The impedance drop seen after stimulation was a reproducible phenomenon over the 12 weeks of chronic implantation. Over this period, the time of implantation did not affect the degree of impedance reduction (Figure 8).

The EIS data comparison before and after stimulation showed consistency with time at $1 \mathrm{kHz}$ in all subjects. The mean impedance of contacts at $1 \mathrm{kHz}$ stimulated for 10 min drops to values below $7 \mathrm{kOhm}$, i.e. a drop of $\sim 60 \%$. There is very little variability between different electrode sites.

As the phenomenon was very reproducible approaching the end of the experiment period, we started investigating the duration of impedance drop. To this aim, at week 9 , before and after a 10 -minute stimulation period, we recorded the impedance every 5 minutes during a 1-hour period without being able to highlight significant impedance recovery (Figure 9). The change of impedance phase and magnitude were characteristic of the effect of electrical stimulation observed during the previous experiments. Additionally, four days after the stimulation session of week12, we tested a short stimulation on rat \#9 with impedance measurements before and after ES. Surprisingly, this period was long enough to come back to an impedance state comparable with other "before stimulation" points. Hence, 4 days corresponds to a partial recovery of impedance. Finally, after the 12-week stimulation protocol, we tried a very short stimulation period with the same charge and charge density. The effect of a 15 -second stimulation was a $40 \%$ impedance drop at $1 \mathrm{kHz}$ (Figure 10).

\section{Biocompatibility}

Post explantation evaluation of strip microelectrodes

Visual inspection of the electrode surface using SEM and Forward Binocular Stereo Microscope revealed only minor surface abrasion in the form of lines and dots. For the most part, electrodes showed integrity in the segmented contacts and the tracks connecting the contacts. There was no visible disruption in the microelectrode structure. Let us note that biological contents (cytoplasm and nucleus) were fixed on the electrode surface (see Figure 11A-C and D).

\section{Evaluation of Surrounding Tissue}

Footprints of the rectangular "skates" below which the strips were glued were not visible in the brain. Remarkably, the strip presented little mechanical resistance to extraction, indicating that adhesion between the strip and encapsulation tissue was marginal. All rats showed a fibrous tissue $(40 \pm 10 \mu \mathrm{m}$ thick) covering the totality of the electrode array in both hemispheres (stimulated and non-stimulated).

In Figure 12, we show a comparison of the reactivity of tissue in contact with parylene/platinum stacking. The positive control (RM-B) presented increased GFAP positive immunostaining compared to the sham (no reaction) and electrode (mild reaction).

\section{Discussion}

The majority of commercial cuff electrodes are made of silicone rubber and platinum-iridium (provided by MicroProbes ${ }^{\circledR}$ (Gaithersburg, USA) or Ardiem Medical ${ }^{\circledR}$ (Pennsylvania, USA)) thus limited by the minimum thickness and resolution of the technology. There is now a larger panel of alternative approaches. J.T. Mortimer and N. Bhadra or X. Navarro et al. described their shapes and characteristics in reference reviews [30][31]. Typically, cuff electrodes are made of 2 to 3 metal rings around the epineurium and most of the further evolutions focused on improving either their selectivity or the signal 
to noise ratio. At least 5 alternatives have been proposed: (1) Flat Interface Nerve Electrodes (FINE) gave promising results even for long-term recordings [32]; (2) thin-film Longitudinal IntraFascicular Electrodes (tfLIFE), and Transverse Intrafascicular Multichannel Electrodes (TIME), whose potential for selective stimulation and recordings was investigated using thin film polyimide substrates [33][34][35]; (3) Carbon nanofibers allowed assessment of issues of being focal and minimally invasive, and having enough stiffness for insertion [36]; (4) Laser-cut metal tracks and contacts embedded in silicone rubber in the transverse and longitudinal section of the nerve in front of selected bundles of fibers is a good compromise between resolution and industrial manufacturing [37], pre-strained silicone sheets with multi-contact (up to 33) which reliability was assessed in dogs for six months [38]; (5) TEENI concept [39] is an alternative approach that tends to promote nerve reconstruction in a tube of biocompatible hydrogel along a network of longitudinal planar electrodes in the case of amputation.

The present study deals with the chronic biocompatibility and reliability of a parylene/platinum multielectrode array that may be embedded in a cuff made of silicone rubber if it meets the reliability and safety criteria. Prior to implantation, the life-time of the electrode manufactured by CEA Leti was estimated to be 4.5 years by accelerated ageing at $67^{\circ} \mathrm{C}$ in aqueous solution (PBS 1X) [18]. As the extrapolation to real conditions raises complex questions, we scheduled in-vivo tests on small animals for a 12-week period, which corresponds to the duration of long-term biocompatibility standards (ISO 10993-6). Rodger et al reported a durability of at least 6 months with another Parylene/platinum electrode array [40]. We know from our previous experience that even if parylene is very stable in vivo, cracks and delamination are likely to occur at the parylene/parylene or parylene/metal interface due to its poor adhesion and limited elongation [17][41].

Such thin film micro electrode arrays could be useful for eortical stimulation / recording in applications requiring high density of electrodes or closer contact with the grey matter for instance the future generation of WIMAGINE®, the CEA Leti wireless recording device for BCI [42], enabling better brain control of effectors such as exoskeleton for quadriplegic or enabling more accurate recording for epilepsy study. Other research groups and industrials (Cortec, Wise or Ripple) already published partial validation of passive [43] or active [44] cortical thin-film electrode arrays. The evolution of resolution depending on the implantation techniques [45][46][47] or the brain tissue response are also very well documented for rats [48], cats [49] or non-human primates [50].

In their final use as cuff electrodes, we shall customize the arrays into a cylindrical shape. However, considering the small diameter of peripheral nerves of rats and the risks associated with a percutaneous connector, we simplified the problem to planar electrode arrays on the rats' dura-mater. In this way, we focused on two questions: (1) tissue reaction towards segmented platinum electrodes (2) the effects of clinically relevant electrical stimulation using the prototype electrode array.

\section{Chronic behavior of the implanted electrodes}

To confirm the preliminary results obtained in PBS-1X, we implanted a group of animals to assess the chronic behavior and reliability of the electrodes under electrical stimulation during the 12-week protocol. On a daily observation, no clinical problem was reported. Additionally, we observed a decrease in the impedance on a medium frequency window with a remarkable consistency.

Even if the implantation is chronic ( $>30$ days), the electrical stimulation is intermittent since it is applied during 10 minutes once a week. Indeed, to apply a chronic stimulation, one should either miniaturize a wearable pulse generator and its power supply or use a larger animal model such as pig, sheep or

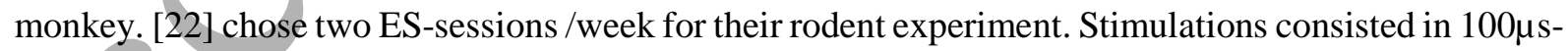
long biphasic phases at 50 pulses/s during 1s whereas [51] respectively 2-hour ES sessions every day during 5 days on guinea pig with $100 \mu$ s-long biphasic phases, 250 pulses/s). For non-human primate, [52] chose a $90 \mu$ s pulse repeated at $130 \mathrm{~Hz}$ one hour a day during 100days. 
With our stimulation pattern, we tried to optimize neuronal recruitment and reduced tissue damage and electrode corrosion. The maximum charge injection per pulse $\left(18.3 \mu \mathrm{C} / \mathrm{mm}^{2}\right)$ is below the maximum quantified in PBS $\left(20.2 \mu \mathrm{C} / \mathrm{cm}^{2}\right)$ for sputtered platinum and the limit of $50 \mu \mathrm{C} / \mathrm{cm}^{2}$ given by Rose and Robblee [28]. If the charge injection request is too high for platinum contact, dissolution is likely to occur. Based on the voltage curve of (Figure 4B), we may say that the charge exchange at the electrode surface only involved reversible platinum oxides since we did not see any inflexion point related to the initiation of new electrochemical systems [53]. We chose active biphasic stimulation consisting of a small anodic stimulation (anodic first $(\mathrm{AF}),(0.264 \mathrm{~mA}$, duration $1.36 \mathrm{~ms})$. This is much less than the $225 \mu \mathrm{C} / \mathrm{cm}^{2}$ estimated for a $1 \mathrm{~ms}$ pulse in the AF mode [28], keeping in mind that short pulses reduce the maximum charge density. Moreover as oxide formation/reduction reactions do not occur during cathodic first (CF) pulsing, and since hydride reactions do not occur during AF pulsing, imposing an anodic bias to the cathodic pulse is a way to take benefit of both [28][54]. Finally, considering two pulses per phase, we are still below the conservative limit of $\mathrm{k}=1.5$.

A stable and reliable electrode interface is mandatory for a chronic stimulating or recording device, either for peripheral or central nervous system modulation or recording. After explantation and cleaning, we did not observe major damage to the contact (Figure 11). We attribute minor cracks to mechanical stresses in the titanium adhesion layer, since we also observed some of these on non-implanted electrodes and to excessive mechanical constraints during the manipulation of the sample during cleaning or preparation for the SEM analysis. We saw no evidence of corrosion or dissolution, but SEM is much less sensitive to quantification of platinum traces than atomic absorption rate is. Alternatively, the assessment of the Pt-O content would be of interest for a new campaign of experiments.

As expected, the effect of long-term implantation in the non-stimulating electrodes was an increase of the impedance during the first 2 weeks of implantation, followed by stabilization at a lower level. This level is nevertheless higher than the initial state prior to implantation (see Figure 7C), especially in the medium range frequencies. This had already been attributed to biotic and abiotic phenomena [55]. Our observations are consistent with those described in the literature about ECoG electrode arrays [56][57][58], where encapsulation generates an increase of impedance and attenuation of the signal, which is prejudicial for implantable BCI devices above $120 \mathrm{~Hz}$ [59]. This type of change in impedance in-vivo is explained by thickening of the duramater and growth of a fibrotic encapsulation of the electrodes, increasing the impedance of the recording with a serial resistance [60]. Additionally, the timescale of impedance evolution is consistent with intraparenchymal tissue reactions [22] and results on micro ECoG grid in the retina [61], in which tissue reaction and encapsulation reach their heights after 3 weeks, and then stabilize.

The overall trend of the impedance data, and in particular the impedance drop after electrical stimulation, matches the observations of other investigators using deep brain stimulation probes [52] or intracortical rings [22], silicon probes [51]. In Lempka et al's study, clinically relevant electrical stimulation in a macaque model produced a rapid decrease in electrode impedance with broad changes in the surrounding tissues. [62] and [63] also showed impedance drops immediately after electrical stimulation either during in-vitro experiments or in preclinical studies of cochlear electrodes connected to clinically-relevant biphasic current pulse stimulators. Weiland [51] and Wang [22] also reported an impedance drop after electrical stimulation, using intraparenchymal electrodes based on silicon or metallic needles with microelectrodes $\left(400 \mu \mathrm{m}^{2}\right.$ and $\left.1600 \mu \mathrm{m}^{2}\right)$ or annular rings $\left(7854 \mu \mathrm{m}^{2}\right.$ and $\left.15707 \mu \mathrm{m}^{2}\right)$. The poststimulation impedance at mid-range frequencies $(100-100 \mathrm{k}) \mathrm{Hz}$ consistently and significantly decreased relative to pre-stimulation levels for about 24 and 3 hours respectively. These results are consistent with oùr data: each time we applied ES, we observed a significant impedance drop and we reported this phenomenon during the 12-week period of chronic implantation.

The other significant effect was that the baseline impedance between stimulation trials did not increase, as would have been expected because of encapsulation. As seen in the contralateral hemisphere, foreign body reactions produce an increase in the impedance that usually stabilizes around 2 weeks postoperation. 
It is difficult to explain exactly what happened during electrical stimulation. According to Wang et al. [22], ES may have repulsed the dura or broken a thin encapsulation sheath creating temporary conditions in front of the contact close to those of the preimplantation tests in PBS or CSF media. It is possible that in our study a smaller tissue encapsulation, compared to intraparenchymal electrodes and different electrical stimulation parameters, could explain why at each session from weeks 6 to 12 we still reported impedance drops after electrical stimulation. According to our data, the impedance versus time is an increasing non-monotonous function but impedances dropped down to a remarkably stable state after each stimulation window. The impedance recovery is nearly visible after 1 hour and lasts for up to 3 days (Figure 9 and Figure 10).

An additional possibility is the electroporation of the surrounding tissue, in which membrane permeability increases as a result of the application of an electrical field [62]. In-vitro and in-vivo studies have shown that a cell layer in contact with the electrode surface can increase the formation of pores in the cell membrane and affects the tight junction in the overlying tissue, producing the reduction in impedance and possibly, at higher intensity, cell death [64], [65].

\section{Biocompatibility}

This was a first step toward the validation of implantable multiple contact cuff electrodes based on such technology. Despite the complexity of the manufacture of the multi-contact strip, technical failures are rare. Nevertheless, as time went by (after week \#2 or \#6), the impedance magnitude of six out of seven of the E4 contacts dramatically increased ( $>200 \mathrm{kOhm}$ instead of a few $\mathrm{kOhm}$ at $1 \mathrm{kHz}$ ). We assume there was an interconnection issue and suggest packaging modification if we were to manufacture another batch of electrode arrays. Fortunately, we only faced this issue on contacts of the control array.

Tissue reaction to electrode materials has been widely reported in the literature. Work carried out by Stensaas et al., showed no adverse histological reaction to platinum, gold and tungsten when implanted more than 30 days in cat's cortex [66]. Parylene and polyimide tissue reactions have also been extensively studied and the findings are also only a minimal reaction similar to that of pure platinum inside cortical surfaces [67]. In the same study, after 16 weeks all neurons beneath the implant appeared to be normal. Additionally, [14] investigated the influence of the geometry of the parylene substrate (solid, mesh, fenestrated) using impedance spectroscopy and in-vivo imaging in a rat model, highlighting the role of collagen in the tissue sheath. These published findings are consistent with our own findings of minimal tissue reactions in neural tissue compared to positive controls. However, this kind of array always produces either an increase in duramater thickness or simply fibrotic tissue deposition over the surface of electrodes that could potentially reduce transmission and affect long-term efficacy of subdural implant [50]. As explained above, the deposition of fibrous tissue $(40 \pm 10 \mu \mathrm{m}$ thick) in all subjects, contiguous to the electrode active surface (either stimulated or not), possibly helps to explain the steady increase of impedance in the non-stimulated electrodes during the 12 weeks of experimental protocols. Adding quantification of the cell types as done by [50] (on Rhesus macaque subdurally implanted with PMT Corp. electrode arrays) would have been interesting and should be performed within the next studies.

We had no problem extracting the electrode array from the cortex. This finding indicates a minimum adhesion between tissue and electrode interface. The risk of tissue adhesion is reduced by the lack of porosity and high degree of smoothness [68], which is consistent with thin film depositions (parylene and platinum) performed over polished silicon wafers. It should be remembered that during the course of the project, commercial devices such as the Second Sight $(\subset$ visual prosthesis based on polyimide were FDA approved. Moreover, a recent comparison of the foreign body reaction (FBR) gave an advantage to polyimide compared to parylene $\mathrm{C}$ for intra-fascicular electrodes due to a higher hydrophobicity and higher stability in-vivo [69]. Consequently, if we were to start another production run, we would rather choose the polyimide option, at least for its outstanding thermal resistance, drastically easing the manufacturing process and improving adhesion. 


\section{Conclusions}

The starting point of this study was to assess the reliability and biocompatibility of microelectrode arrays made of thin film parylene and metal tracks. A previous accelerated ageing campaign gave us the confidence that the electrodes could undergo long-term implantation including electrical stimulation. For the sake of simplicity, we reduced the initial schedule of conditions of a cuff electrode to a planar array implanted epidurally. The follow-up consisted of weekly impedance spectroscopy before and after a 10-minute electrical stimulation period. We highlight the following results:

1. The electrode arrays manufactured by CEA Leti in cortical situation underwent the 3-month implantation and tissue response although qualitative shows encouraging results in terms of biocompatibility;

2. This study brings new evidence of the effect of Electrical Stimulation on contact impedance. We report a systematic and significant drop ( $60 \%)$ of impedance at mid-range frequencies after electrical stimulation. Wang et al or Weiland et al reported such drops on smaller timeframes. Here the different stimulation parameters and tissue sheaths may account for the new result.

These results are first validations of this technology for in-vivo microelectrodes. Further preclinical experiments are required with micro-contacts embedded in cuff electrodes to evaluate mechanical resistance to cylindrical deformation and long-term quality of the contact between cuff electrodes and nerves. In addition, continuous chronic stimulation should be investigated using a larger animal model or a portable stimulation system to investigate the feasibility of a clinical use.

\section{Acknowledgements}

This study is part of a consortium working within the INTENSE project, partially supported by BPI France, within the 'Investments for the Future' program in France.

F. Bottausci, N. Verplanck, C. Pudda, F. Baleras, G. Grenot, M.C. Gentet (CEA Leti DBTS) designed and manufactured the parylene/platinum electrode arrays and the instrumentation set up.

M. D'Orchymont (CEA Leti CLINATEC) performed the surgery on rodents.

\section{REFERENCES}

[1] A. Ritaccio et al., "Proceedings of the Second International Workshop on Advances in Electrocorticography," Epilepsy Behav., vol. 22, no. 4, pp. 641-650, Dec. 2011.

[2] K. N. Browning, S. Verheijden, and G. E. Boeckxstaens, "The Vagus Nerve in Appetite Regulation, Mood, and Intestinal Inflammation," Gastroenterology, vol. 152, no. 4, pp. 730-744, 2017.

[3] H. H. O. Müller, S. Moeller, C. Lücke, A. P. Lam, N. Braun, and A. Philipsen, "Vagus Nerve Stimulation (VNS) and Other Augmentation Strategies for Therapy-Resistant Depression (TRD): Review of the Evidence and Clinical Advice for Use," Front. Neurosci., vol. 12, Apr. 2018.

[4] D. Guiraud et al., "Vagus nerve stimulation: state of the art of stimulation and recording strategies to address autonomic function neuromodulation," J. Neural Eng., vol. 13, no. 4, p. 041002, 2016.

[5] M. Dali et al., "Model baséd optimal multipolar stimulation without a priori knowledge of nerve structure: application to vagus nerve stimulation," J. Neural Eng., vol. 15, no. 4, p. 046018, 2018.

[6] H. M. Romero-Ugalde et al., "Closed-Loop Vagus Nerve Stimulation Based on State Transition Models," IEEE Trans. Biomed. Eng., vol. 65, no. 7, pp. 1630-1638, Jul. 2018.

[7] D. T. T. Plachta, N. Espinosa, M. Gierthmuehlen, O. Cota, T. C. Herrera, and T. Stieglitz, "Detection of baroreceptor activity in rat vagal nerve recording using a multi-channel cuffelectrode and real-time coherent averaging," in 2012 Annual International Conference of the IEEE Engineering in Medicine and Biology Society, 2012, pp. 3416-3419.

[8] S. Bonnet et al., "Selective ENG recordings using a multi-contact cuff electrode," in 2013 6th International IEEE/EMBS Conference on Neural Engineering (NER), 2013, pp. 923-926. 
[9] C. Hassler, T. Boretius, and T. Stieglitz, "Polymers for neural implants," J. Polym. Sci. Part B Polym. Phys., vol. 49, no. 1, pp. 18-33, 2011.

[10] P. J. Rousche, D. S. Pellinen, D. P. Pivin, J. C. Williams, R. J. Vetter, and D. R. Kipke, "Flexible polyimide-based intracortical electrode arrays with bioactive capability," IEEE Trans. Biomed. Eng., vol. 48, no. 3, pp. 361-371, Mar. 2001.

[11] K. C. Cheung, P. Renaud, H. Tanila, and K. Djupsund, "Flexible polyimide microelectrode array for in vivo recordings and current source density analysis," Biosens. Bioelectron., vol. 22, no. 8, pp. 1783-1790, Mar. 2007.

[12] B. Rubehn and T. Stieglitz, "In vitro evaluation of the long-term stability of polyimide as a material for neural implants," Biomaterials, vol. 31, no. 13, pp. 3449-3458, May 2010.

[13] D. S. Lee et al., "Comparison of in vivo biocompatibilities between parylene-C and polydimethylsiloxane for implantable microelectronic devices," Bull. Mater. Sci., vol. 36, no. 6, pp. 1127-1132, 2013.

[14] A. A. Schendel et al., "The effect of micro-ECoG substrate footprint on the meningeal tissue response," J. Neural Eng., vol. 11, no. 4, p. 046011, Aug. 2014.

[15] S. Takeuchi, D. Ziegler, Y. Yoshida, K. Mabuchi, and T. Suzuki, "Parylene flexible neural probes integrated with microfluidic channels," Lab. Chip, vol. 5, no. 5, pp. 519-523, 2005.

[16] V. Castagnola et al., "Parylene-based flexible neural probes with PEDOT coated surface for brain stimulation and recording," Biosens. Bioelectron., vol. 67, pp. 450-457, May 2015.

[17] E. M. Schmidt, J. S. McIntosh, and M. J. Bak, "Long-term implants of Parylene-C coated microelectrodes," Med. Biol. Eng. Comput., vol. 26, no. 1, pp. 96-101, 1988.

[18] F. Bottausci et al., "New sectorized implantable microelectrode fabrication, packaging and ageing for neural sensing and stimulation," in 2015 7th International IEEE/EMBS Conference on Neural Engineering (NER), 2015, pp. 466-469.

[19] D. H. Szarowski et al., "Brain responses to micro-machined silicon devices," Brain Res., vol. 983, no. 1-2, pp. 23-35, Sep. 2003.

[20] D. R. Merrill, M. Bikson, and J. G. R. Jefferys, "Electrical stimulation of excitable tissue: design of efficacious and safe protocols," J. Neurosci. Methods, vol. 141, no. 2, pp. 171-198, Feb. 2005.

[21] D. McCreery, V. Pikov, and P. R. Troyk, "Neuronal loss due to prolonged controlled-current stimulation with chronically implanted microelectrodes in the cat cerebral cortex," J. Neural Eng., vol. 7, no. 3, p. 036005, 2010.

[22] C. Wang, E. Brunton, S. Haghgooie, K. Cassells, A. Lowery, and R. Rajan, "Characteristics of electrode impedance and stimulation efficacy of a chronic cortical implant using novel annulus electrodes in rat motor cortex," J. Neural Eng., vol. 10, no. 4, p. 046010, 2013.

[23] D. Andreu, D. Guiraud, and G. Souquet, "A distributed architecture for activating the peripheral nervous system," J. Neural Eng., vol. 6, no. 2, p. 026001 , Feb. 2009.

[24] P. Maciejasz et al., "Delaying discharge after the stimulus significantly decreases muscle activation thresholds with small impact on the selectivity: an in vivo study using TIME," Med. Biol. Eng. Comput., vol. 53, no. 4, pp. 371-379, Apr. 2015.

[25] N. de N. Donaldson and P. E. K. Donaldson, "When are actively balanced biphasic ('Lilly') stimulating pulses necessary in a neurological prosthesis? II pH changes; noxious products; electrode corrosion; discussion," Med. Biol. Eng. Comput., vol. 24, no. 1, pp. 50-56, 1986.

[26] S. F. Cogan, "Neural Stimulation and Recording Electrodes," Annu. Rev. Biomed. Eng., vol. 10, no. 1, pp. 275-309, 2008.

[27] L. S. Robblee, J. McHardy, W. F. Agnew, and L. A. Bullara, "Electrical stimulation with Pt electrodes. VII. Dissolution of Pt electrodes during electrical stimulation of the cat cerebral cortex," J. Neurosci. Methods, vol. 9, no. 4, pp. 301-308, Dec. 1983.

[28] T. L. Rose and L. S. Robblee, "Electrical stimulation with Pt electrodes. VIII. Electrochemically safe charge injection limits with $0.2 \mathrm{~ms}$ pulses (neuronal application)," IEEE Trans. Biomed. Eng., vol. 37, no. 11, pp. 1118-1120, Nov. 1990.

[29] P. Romanelli et al., "A novel neural prosthesis providing long-term electrocorticography recording and cortical stimulation for epilepsy and brain-computer interface," J. Neurosurg., pp. 1-14, May 2018.

[30] K. W. Horch and G. S. Dhillon, Neuroprosthetics: theory and practice. New Jersey: World Scientific, 2004. 
[31] X. Navarro, T. B. Krueger, N. Lago, S. Micera, T. Stieglitz, and P. Dario, "A critical review of interfaces with the peripheral nervous system for the control of neuroprostheses and hybrid bionic systems," J. Peripher. Nerv. Syst., vol. 10, no. 3, pp. 229-258, Sep. 2005.

[32] Y. M. Dweiri, T. E. Eggers, L. E. Gonzalez-Reyes, J. Drain, G. A. McCallum, and D. M. Durand, "Stable Detection of Movement Intent From Peripheral Nerves: Chronic Study in Dogs," Proc. IEEE, vol. 105, no. 1, pp. 50-65, Jan. 2017.

[33] J. Badia, T. Boretius, D. Andreu, C. Azevedo-Coste, T. Stieglitz, and X. Navarro, "Comparative analysis of transverse intrafascicular multichannel, longitudinal intrafascicular and multipolar cuff electrodes for the selective stimulation of nerve fascicles," J. Neural Eng., vol. 8, no. 3, p. 036023 , May 2011.

[34] T. Boretius et al., "A transverse intrafascicular multichannel electrode (TIME) to interface with the peripheral nerve," Biosens. Bioelectron., vol. 26, no. 1, pp. 62-69, Sep. 2010.

[35] J. Badia, T. Boretius, A. Pascual-Font, E. Udina, T. Stieglitz, and X. Navarro, "Biocompatibility of Chronically Implanted Transverse Intrafascicular Multichannel Electrode (TIME) in the Rat Sciatic Nerve," IEEE Trans. Biomed. Eng., vol. 58, no. 8, pp. 2324-2332, Aug. 2011.

[36] P. R. Patel et al., "Chronic in vivo stability assessment of carbon fiber microelectrode arrays," $J$. Neural Eng., vol. 13, no. 6, p. 066002, 2016.

[37] M. Mueller, N. de la Oliva, J. del Valle, I. Delgado Martinez, X. Navarro, and T. Stieglitz, "Rapid prototyping of flexible intrafascicular electrode arrays by picosecond laser structuring," J. Neural Eng., Jul. 2017.

[38] J. Rozman, B. Zorko, M. Bunc, U. Mikac, and E. Tegou, "Recording of ENGs from the nerves innervating the pancreas of a dog during the intravenous glucose tolerance test," Physiol. Meas., vol. 23, no. 4, p. 695, 2002.

[39] B. S. Spearman et al., "Tissue-Engineered Peripheral Nerve Interfaces," Adv. Funct. Mater., p. n/a-n/a.

[40] D. Rodger et al., "Flexible parylene-based multielectrode array technology for high-density neural stimulation and recording," Sens. Actuators B Chem., vol. 132, no. 2, pp. 449-460, Jun. 2008.

[41] J. C. Barrese et al., "Failure mode analysis of silicon-based intracortical microelectrode arrays in non-human primates," J. Neural Eng., vol. 10, no. 6, p. 066014, 2013.

[42] C. S. Mestais, G. Charvet, F. Sauter-Starace, M. Foerster, D. Ratel, and A. L. Benabid, "WIMAGINE: wireless 64-channel ECoG recording implant for long term clinical applications.," IEEE Trans. Neural Syst. Rehabil. Eng. Publ. IEEE Eng. Med. Biol. Soc., vol. 23, no. 1, pp. 1021, Jan. 2015.

[43] F. Kohler, T. Stieglitz, and M. Schuettler, "Morphological and electrochemical properties of an explanted PtIr electrode array after 15 months in vivo," in 2014 36th Annual International Conference of the IEEE Engineering in Medicine and Biology Society, 2014, pp. 418-421.

[44] J. Viventi et al., "Flexible, foldable, actively multiplexed, high-density electrode array for mapping brain activity in vivo," Nat. Neurosci., vol. 14, no. 12, pp. 1599-1605, Dec. 2011.

[45] D. T. Bundy et al., "Characterization of the effects of the human dura on macro- and microelectrocorticographic recordings," J. Neural Eng., vol. 11, no. 1, p. 016006, Feb. 2014.

[46] T. J. Oxley et al., "Minimally invasive endovascular stent-electrode array for high-fidelity, chronic recordings of cortical neural activity," Nat. Biotechnol., vol. 34, no. 3, pp. 320-327, Mar. 2016.

[47] E. S. Nurse et al., "Consistency of Long-Term Subdural Electrocorticography in Humans," IEEE Trans. Biomed. Eng., vol. 65, no. 2, pp. 344-352, Feb. 2018.

[48] C. Henle et al., "First long term in vivo study on subdurally implanted Micro-ECoG electrodes, manufactured with a novel laser technology," Biomed. Microdevices, vol. 13, no. 1, pp. 59-68, Feb. 2011.

[49] G. E. Loeb, A. E. Walker, S. Uematsu, and B. W. Konigsmark, "Histological reaction to various conductive and dielectric films chronically implanted in the subdural space," J. Biomed. Mater. Res., vol. 11, no. 2, pp. 195-210, 1977.

[50] A. D. Degenhart et al., "Histological evaluation of a chronically-implanted electrocorticographic electrode grid in a non-human primate," J. Neural Eng., vol. 13, no. 4, p. 046019, Aug. 2016.

[51] J. D. Weiland and D. J. Anderson, "Chronic neural stimulation with thin-film, iridium oxide electrodes," IEEE Trans. Biomed. Eng., vol. 47, no. 7, pp. 911-918, Jul. 2000. 
[52] S. F. Lempka, S. Miocinovic, M. D. Johnson, J. L. Vitek, and C. C. McIntyre, "In vivo impedance spectroscopy of deep brain stimulation electrodes," J. Neural Eng., vol. 6, no. 4, p. 046001, Aug. 2009.

[53] S. C. Mailley, M. Hyland, P. Mailley, J. M. McLaughlin, and E. T. McAdams, "Electrochemical and structural characterizations of electrodeposited iridium oxide thin-film electrodes applied to neurostimulating electrical signal," Mater. Sci. Eng. C, vol. 21, no. 1, pp. 167-175, Sep. 2002.

[54] W. M. Grill and J. T. Mortimer, "Stimulus waveforms for selective neural stimulation," IEEE Eng. Med. Biol. Mag., vol. 14, no. 4, pp. 375-385, Jul. 1995.

[55] A. Prasad et al., "Comprehensive characterization and failure modes of tungsten microwire arrays in chronic neural implants," J. Neural Eng., vol. 9, no. 5, p. 056015, 2012.

[56] P. Fattahi, G. Yang, G. Kim, and M. R. Abidian, "A Review of Organic and Inorganic Biomaterials for Neural Interfaces," Adv. Mater., vol. 26, no. 12, pp. 1846-1885, Mar. 2014.

[57] J. D. Yeager, D. J. Phillips, D. M. Rector, and D. F. Bahr, "Characterization of flexible ECoG electrode arrays for chronic recording in awake rats," J. Neurosci. Methods, vol. 173, no. 2, pp. 279-285, Aug. 2008.

[58] S. Myllymaa et al., "Fabrication and testing of polyimide-based microelectrode arrays for cortical mapping of evoked potentials," Biosens. Bioelectron., vol. 24, no. 10, pp. 3067-3072, Jun. 2009.

[59] D. T. Bundy, M. Pahwa, N. Szrama, and E. C. Leuthardt, "Decoding three-dimensional reaching movements using electrocorticographic signals in humans," J. Neural Eng., vol. 13, no. 2, p. $026021,2016$.

[60] S. Myllymaa, K. Myllymaa, and R. Lappalainen, "Flexible Implantable Thin Film Neural Electrodes," Recent Adv. Biomed. Eng., 2009.

[61] A. Mercanzini, P. Colin, J.-C. Bensadoun, A. Bertsch, and P. Renaud, "In Vivo Electrical Impedance Spectroscopy of Tissue Reaction to Microelectrode Arrays," IEEE Trans. Biomed. Eng., vol. 56, no. 7, pp. 1909-1918, Jul. 2009.

[62] C. Newbold, R. Richardson, R. Millard, P. Seligman, R. Cowan, and R. Shepherd, "Electrical stimulation causes rapid changes in electrode impedance of cell-covered electrodes," J. Neural Eng., vol. 8, no. 3, p. 036029, Jun. 2011.

[63] R. C. de Sauvage, D. L. da Costa, J.-P. Erre, and J.-M. Aran, "Electrical and physiological changes during short-term and chronic electrical stimulation of the normal cochlea," Hear. Res., vol. 110, no. 1-2, pp. 119-134, 1997.

[64] C. Newbold, R. Richardson, C. Q. Huang, D. Milojevic, R. Cowan, and R. Shepherd, “An in vitro model for investigating impedance changes with cell growth and electrical stimulation: implications for cochlear implants," J. Neural Eng., vol. 1, no. 4, pp. 218-227, Dec. 2004.

[65] D. B. McCreery, W. F. Agnew, T. G. H. Yuen, and L. Bullara, "Charge density and charge per phase as cofactors in neural injury induced by electrical stimulation," IEEE Trans. Biomed. Eng., vol. 37, no. 10, pp. 996-1001, 1990.

[66] S. S. Stensaas and L. J. Stensaas, "Histopathological evaluation of materials implanted in the cerebral cortex," Acta Neuropathol. (Berl.), vol. 41, no. 2, pp. 145-155, Feb. 1978.

[67] T. G. Yuen, W. F. Agnew, and L. A. Bullara, "Tissue response to potential neuroprosthetic materials implanted subdurally," Biomaterials, vol. 8, no. 2, pp. 138-141, 1987.

[68] C. M. Sayama, M. Sorour, and R. H. Schmidt, "Dural adhesion to porous cranioplastic implant: A potential safety concern," Surg. Neurol. Int., vol. 5, p. 19, 2014.

[69] N. de la Oliva, M. Mueller, T. Stieglitz, X. Navarro, and J. del Valle, "On the use of Parylene C polymer as substrate for peripheral nerve electrodes," Sci. Rep., vol. 8, no. 1, p. 5965, Apr. 2018. 


\section{FIGURES}

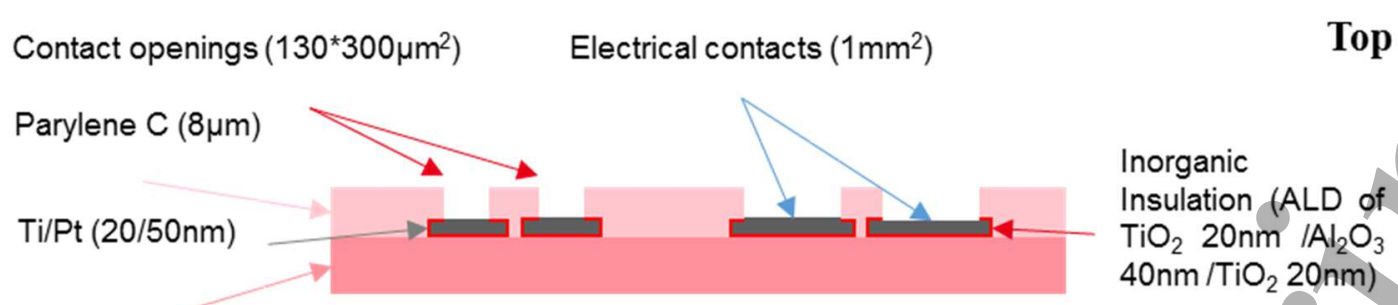

Parylene C $(8 \mu \mathrm{m})$

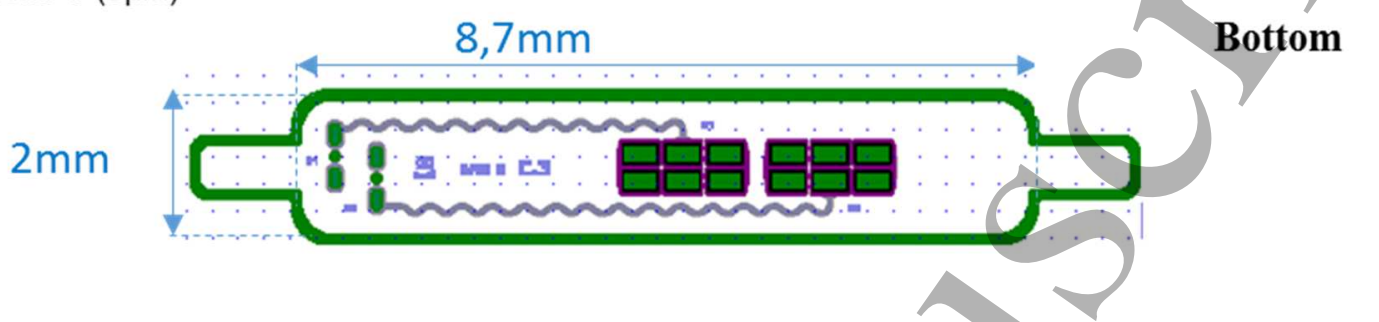

Figure 1 : (Top) Cross view of the electrode grid and (Bottom) Design of the mioroelectrode array (MEA). One strip contains two segmented contacts. Each contact presents six rectangles connected with each other.
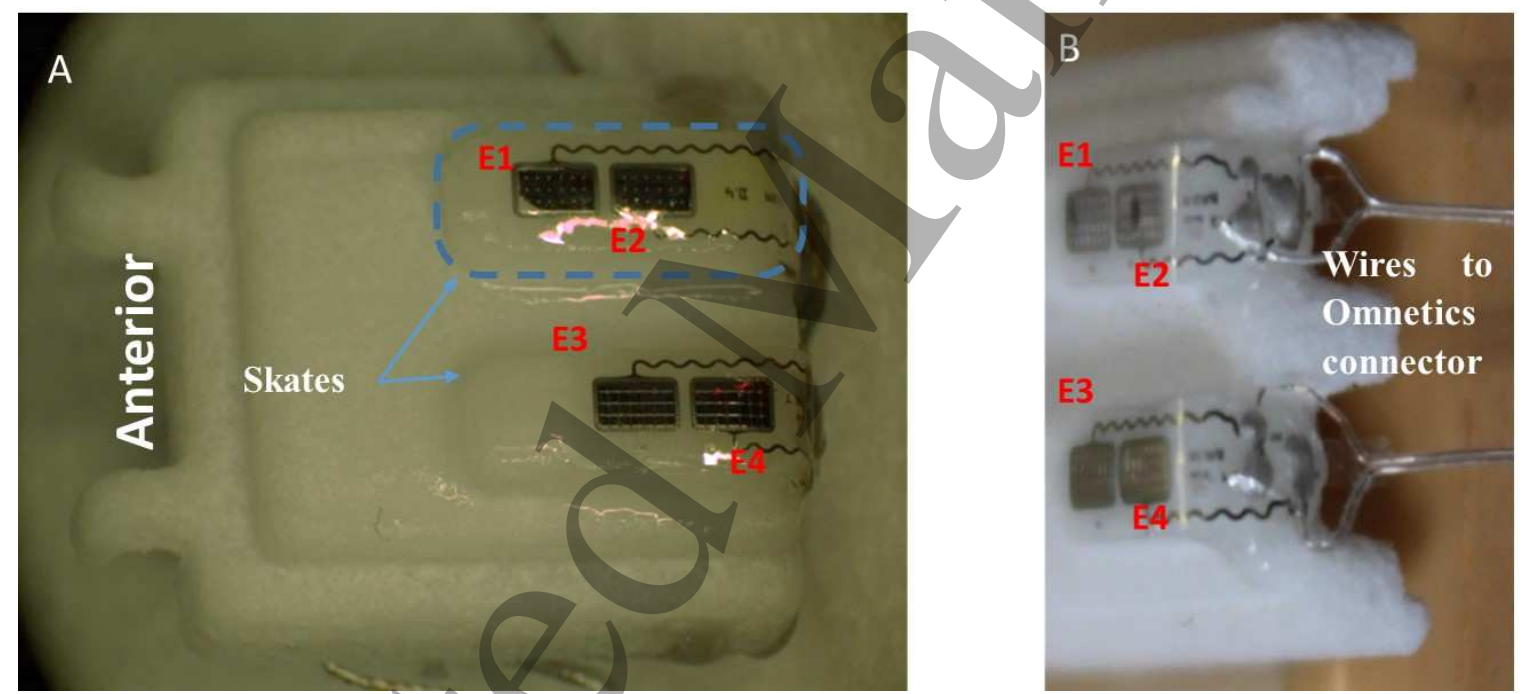

Figure 2: (A) PA2200 platform with two contact strips bottom view, (B) picture with interconnection to the Omnetics connector

45



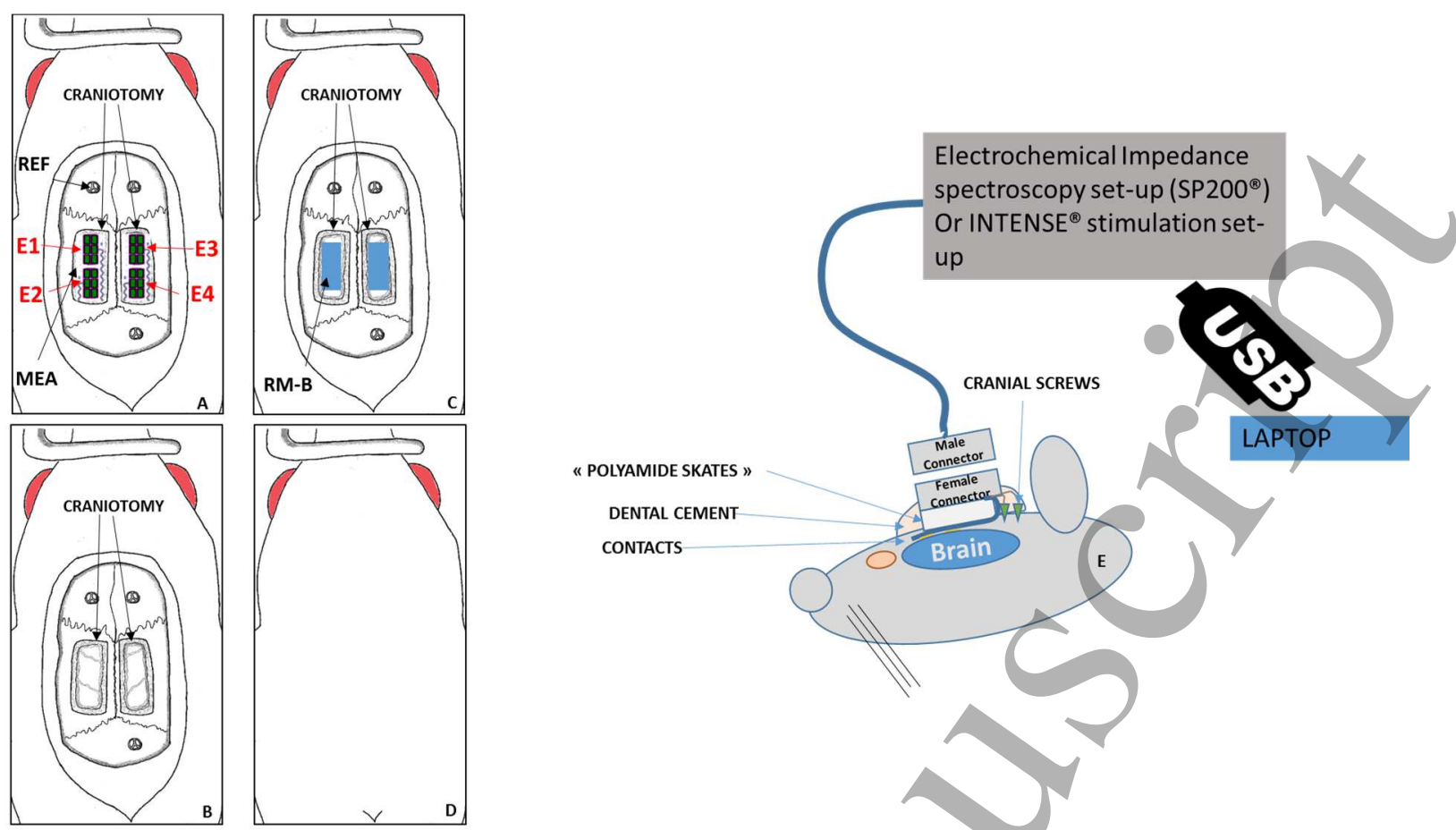

Figure 3: Four group study for electrode evaluation in rodents: (A) Group A: implanted multi segmented contact arrays (B) Sham (group B), (C) Positive control (RM-B). (D) Negative control: no intervention. (E) Cross section of the implantation schema.

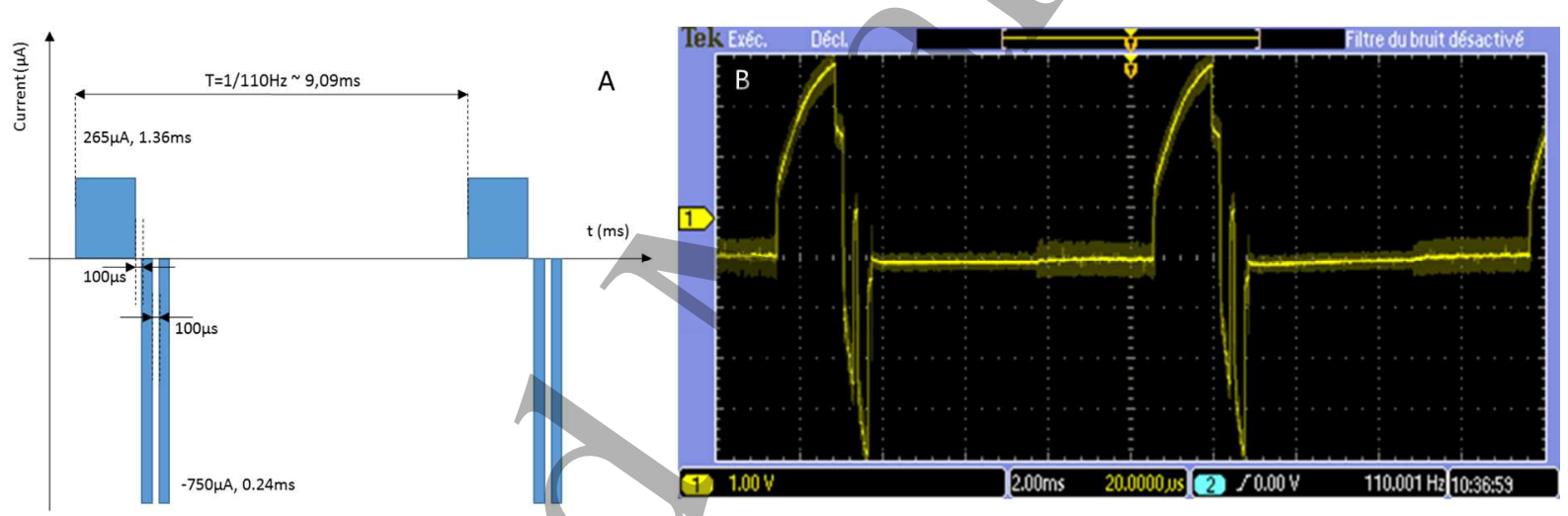

Figure 4: (A) Current controlled stimulation pattern. Charge balanced stimulation was applied in the left hemisphere between the anterior contact (E1) and the posterior contact (E2). (B) Voltage measured between E1 and E2 during stimulation.
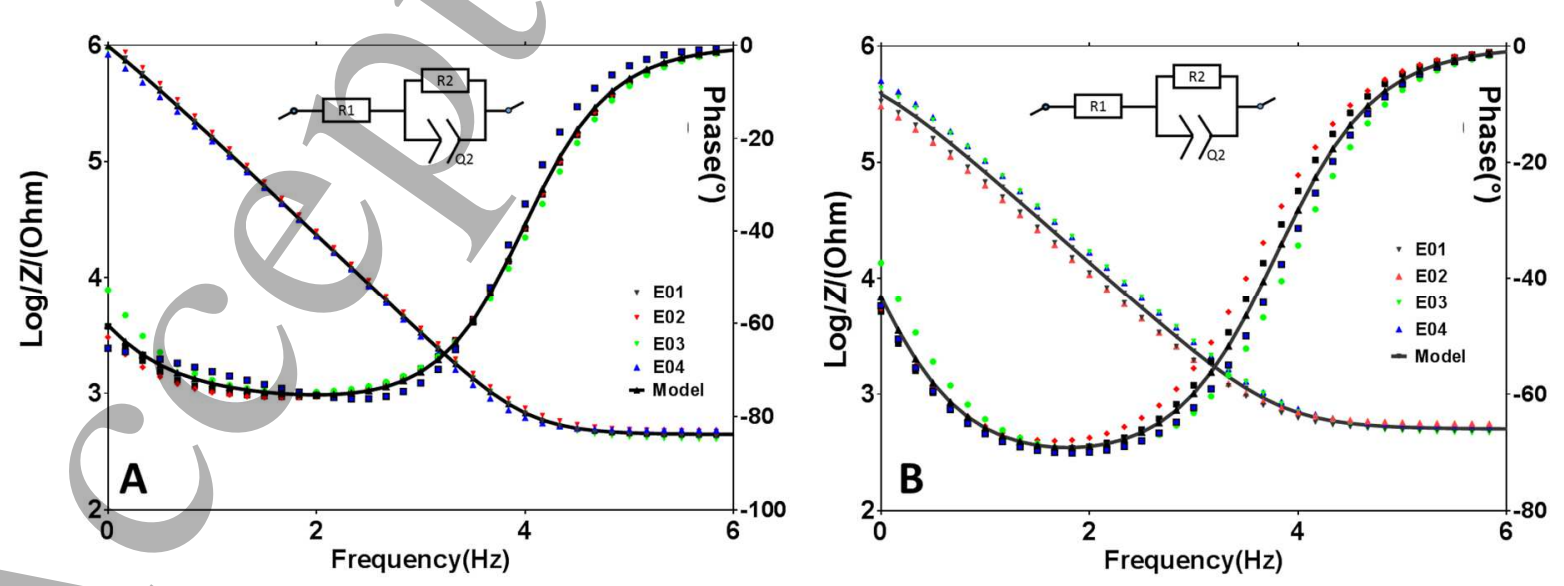
Figure 5: (A) Markers stand for EIS in PBS for each contact of the electrode array BR43 prior to implantation on Rat \#9 compared to the equivalent fitted model (in black) and (B) respectively for BR38 prior to implantation on Rat \#10.
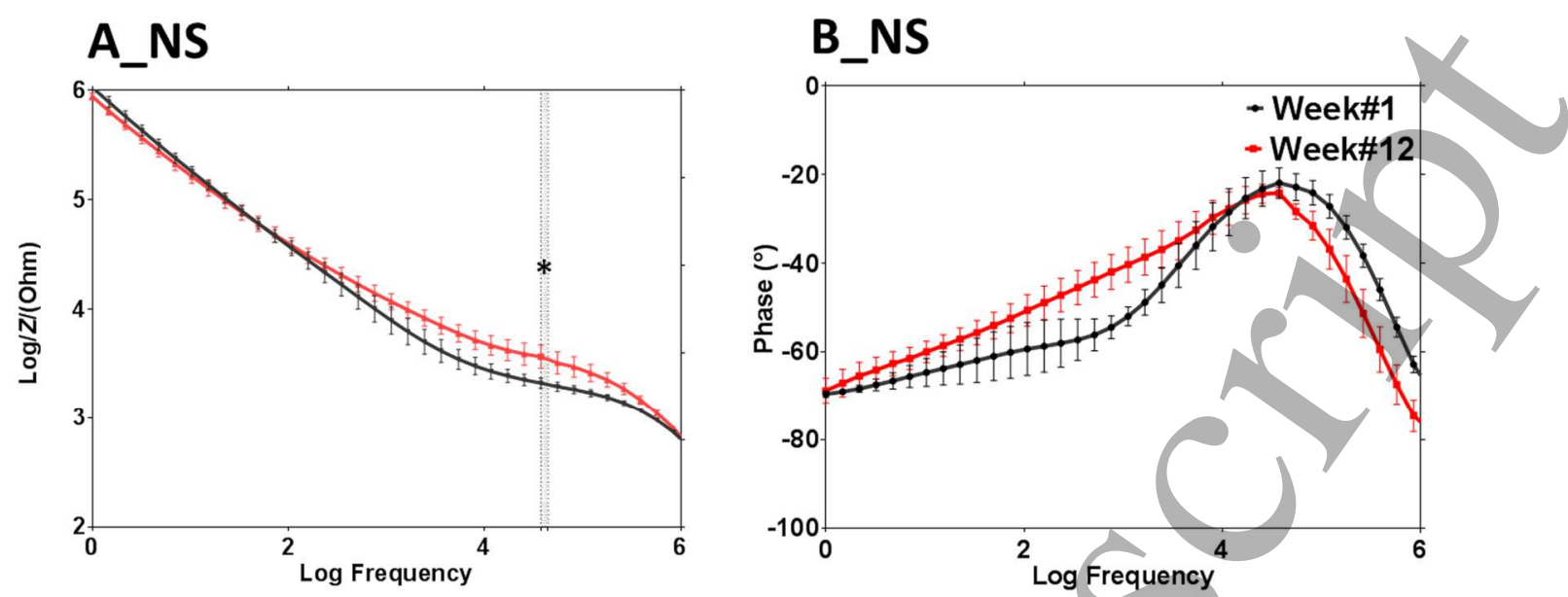

C_NS

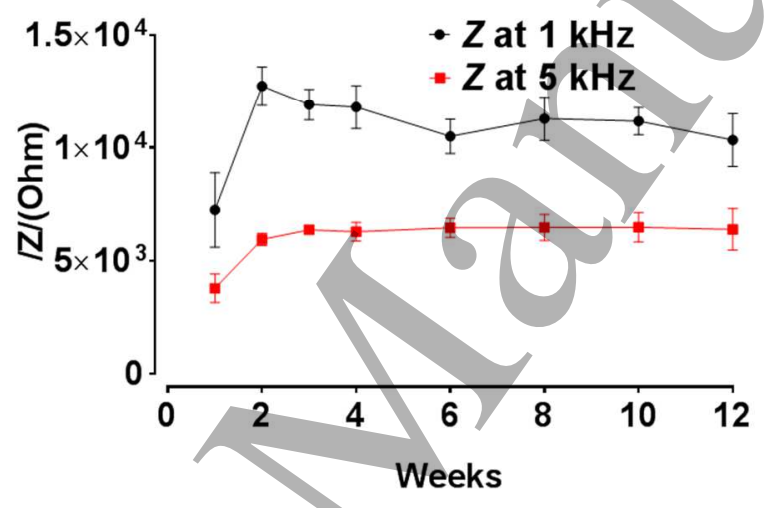

Figure 6: Impedance vs. time of non-stimulated electrodes $(n=7)$. Comparison between initial impedance ( $\left.A \_N S\right)$ and phase (B_NS) after 12 weeks of observation in all frequencies $\left({ }^{*} p<0.05\right)$. (C_NS) Impedance magnitude vs. time of all non-stimulated contacts at $1 \mathrm{kHz}$ and $5 \mathrm{kHz}$. 

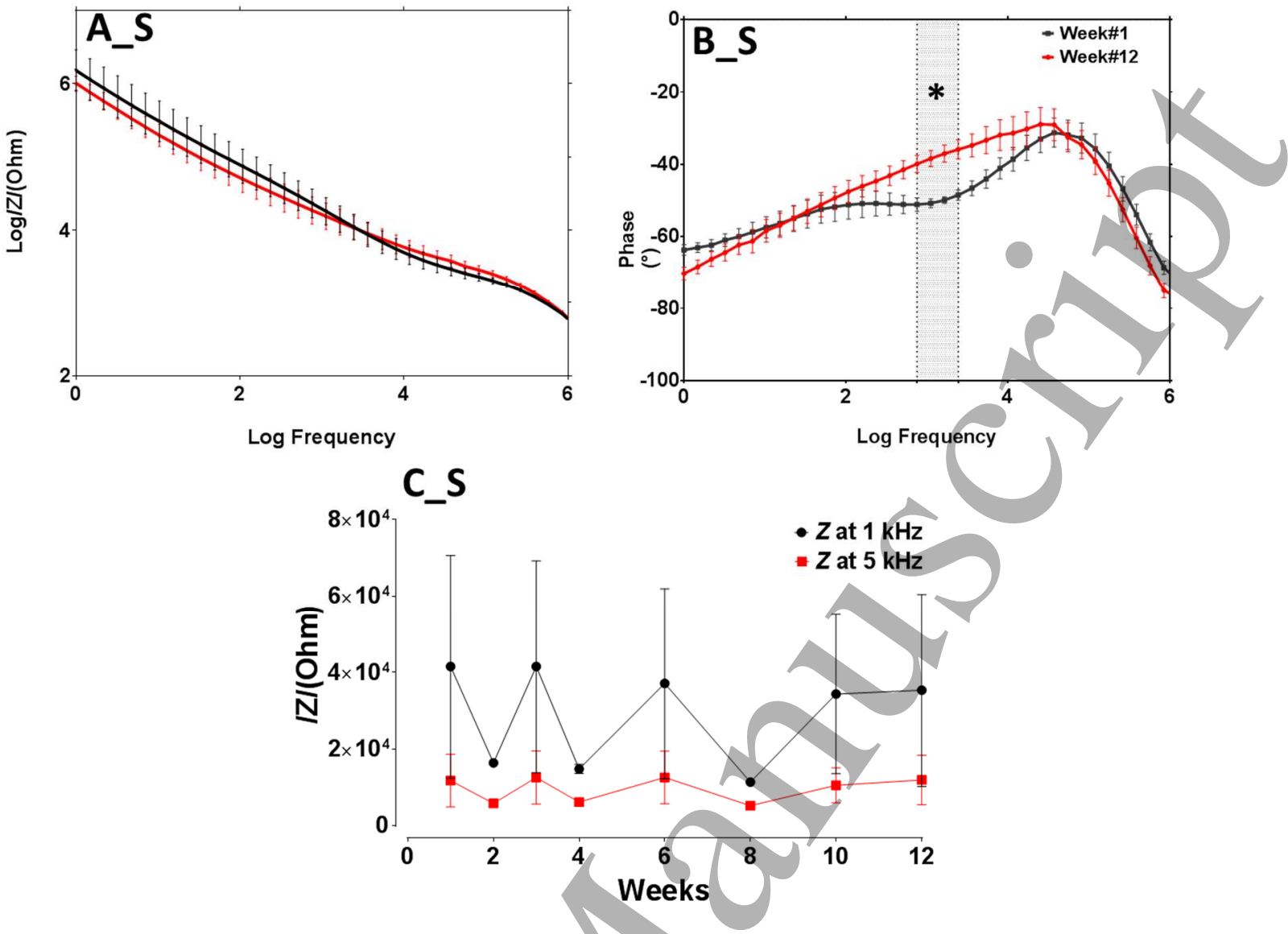

Figure 7 : Evolution of stimulated electrodes vs. time. Impedance and phase were recorded only pre-stimulation to avoid the immediate changes seen in the after stimulation. EIS of all stimulated contacts ( $n=7)$ : comparison between initial impedance ( $A \_S$ ) and phase (B_S) at week one and after 12 weeks of observation in all frequencies. (C_S) Evolution along time of all stimulated contacts at $1 \mathrm{kHz}$ and $5 \mathrm{kHz}$ frequency band. 

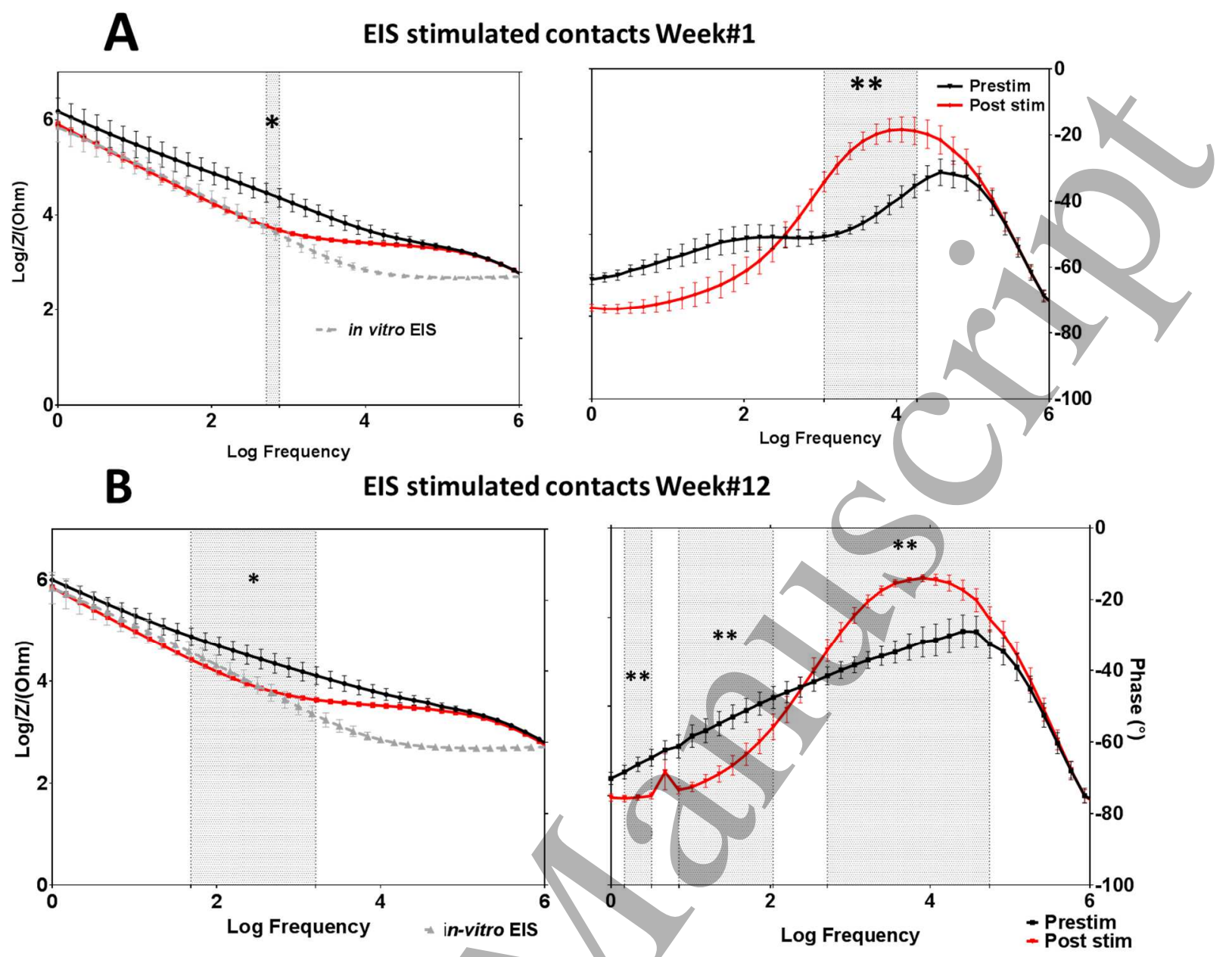

Figure 8 : Evolution of stimulated electrodes pre and post stimulation. (A) EIS of all stimulated electrodes at week\#1 post implantation; (B) EIS of all stimulated electrodes at week\#12 post implantation. There was a significant reduction of impedance post stimulation that was seen from the beginning until the end of the 12-week testing period $\left({ }^{*} p<0.05 ;{ }^{* *} p<0.01\right)$ in both at week \#1 and week\#12. There is also a significant shift in both instances (week\#1 and week\#12) of the phase pre and post stimulation. For comparison, we plot PBS characterization (invitro EIS) in grey 

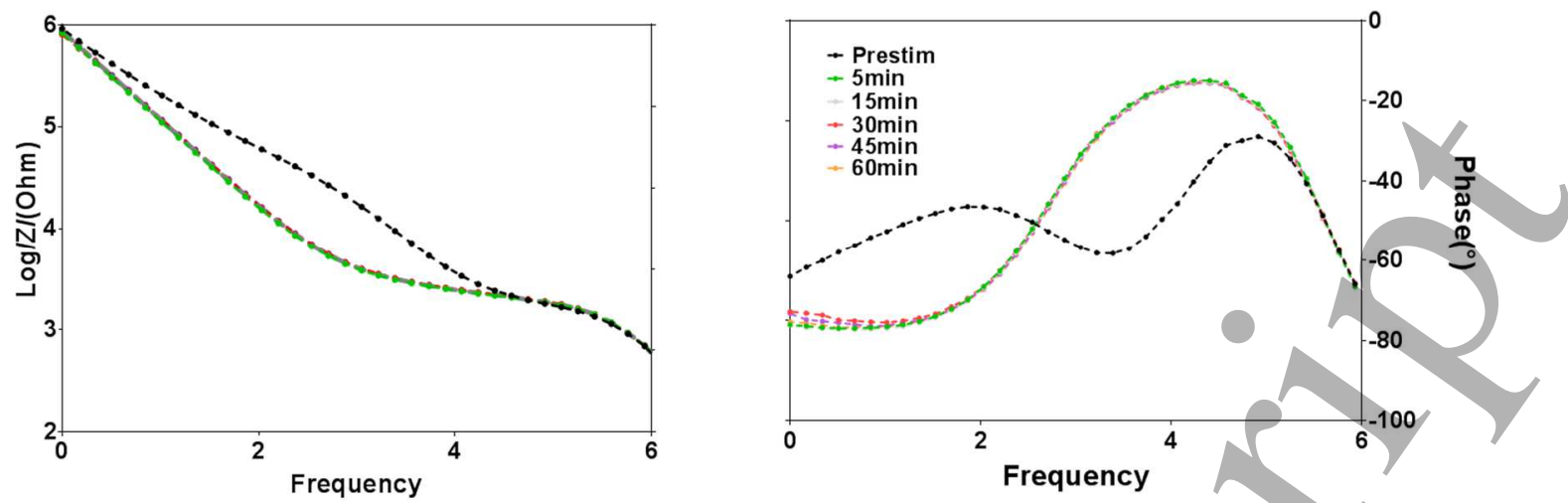

Figure 9 : Evolution of stimulated electrodes pre and post stimulation on rat \#9. Recovery assessment at week 9 over one hour.

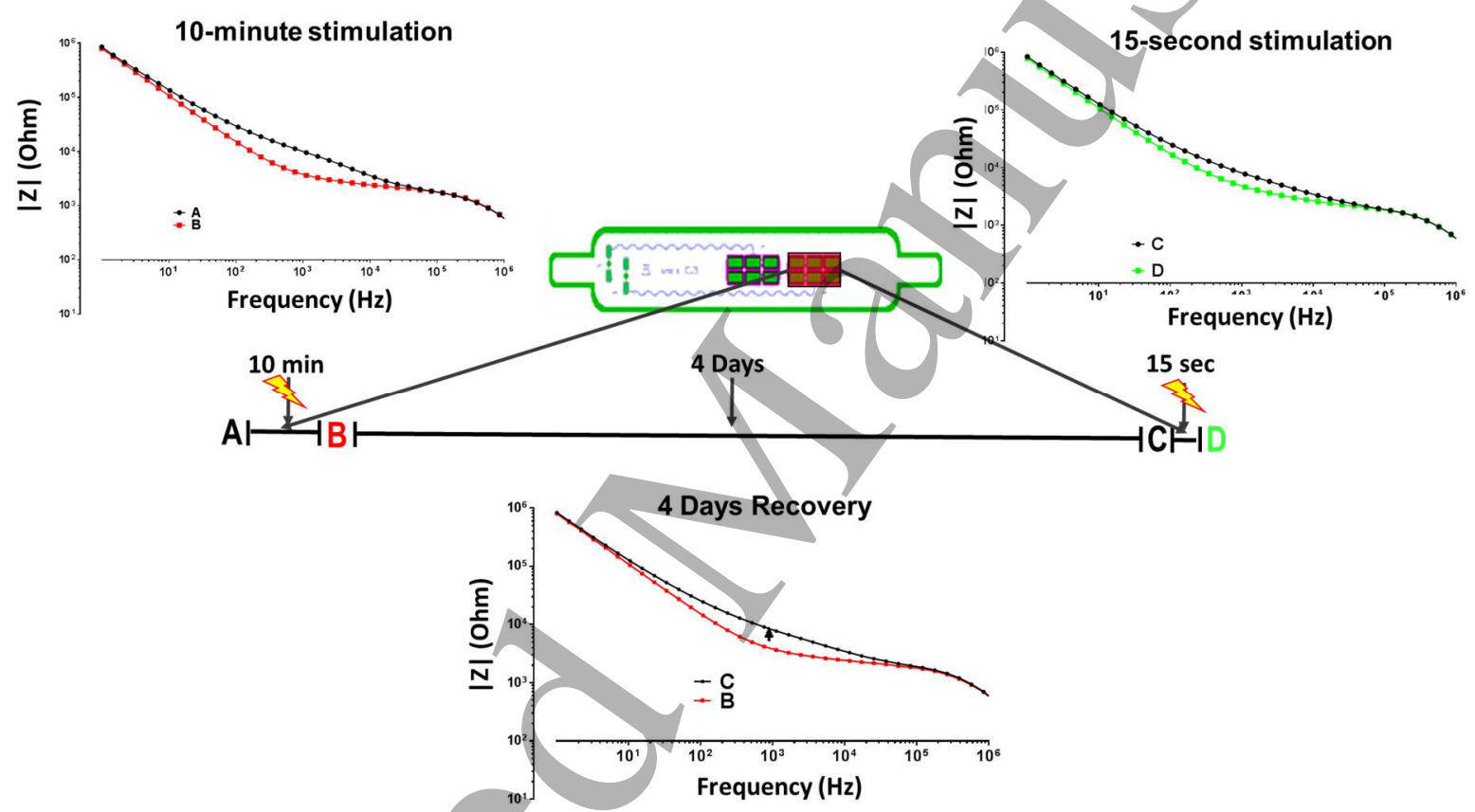

Figure 10 : Example of EIS data after a short stimulation session and spontaneous recovery. At week 12, EIS of E2 contact (A) before and (B) after a 10-minute Electrical Stimulation. Impedance at $1 \mathrm{kHz}$ dropped from to 9.5 to $3.6 \mathrm{kOhm}$ after stimulation. 4-day after 10-minute stimulation shows spontaneous recovery in EIS data. B curve returned to previous baseline values $(C)$ 15-second stimulation triggers $40 \%$ EIS drop at $1 \mathrm{kHz}$. 


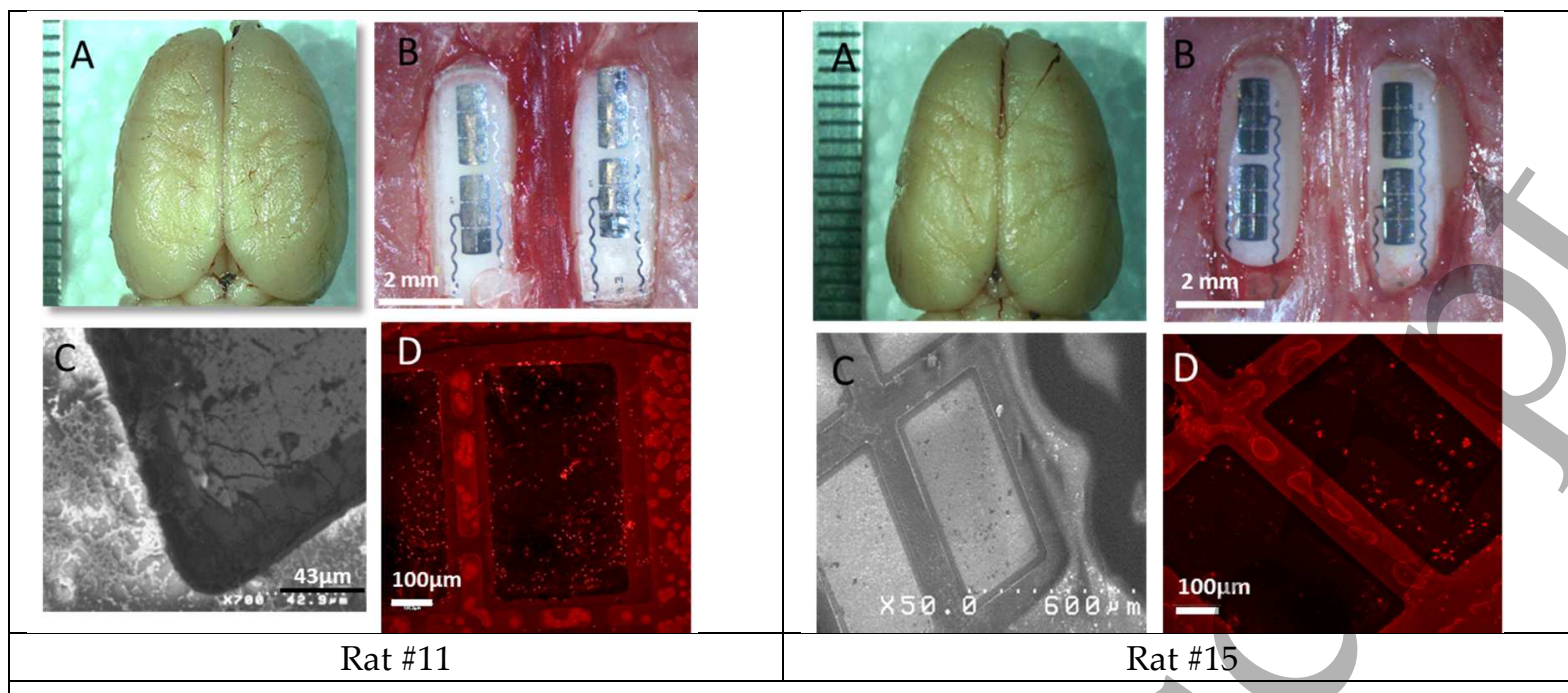

A: anatomical inspection, B: photograph of the strip electrodes in contact with the brain after fibrosis withdrawal, C: SEM picture of a part of contact, D propidium iodide staining to exhibit the cells

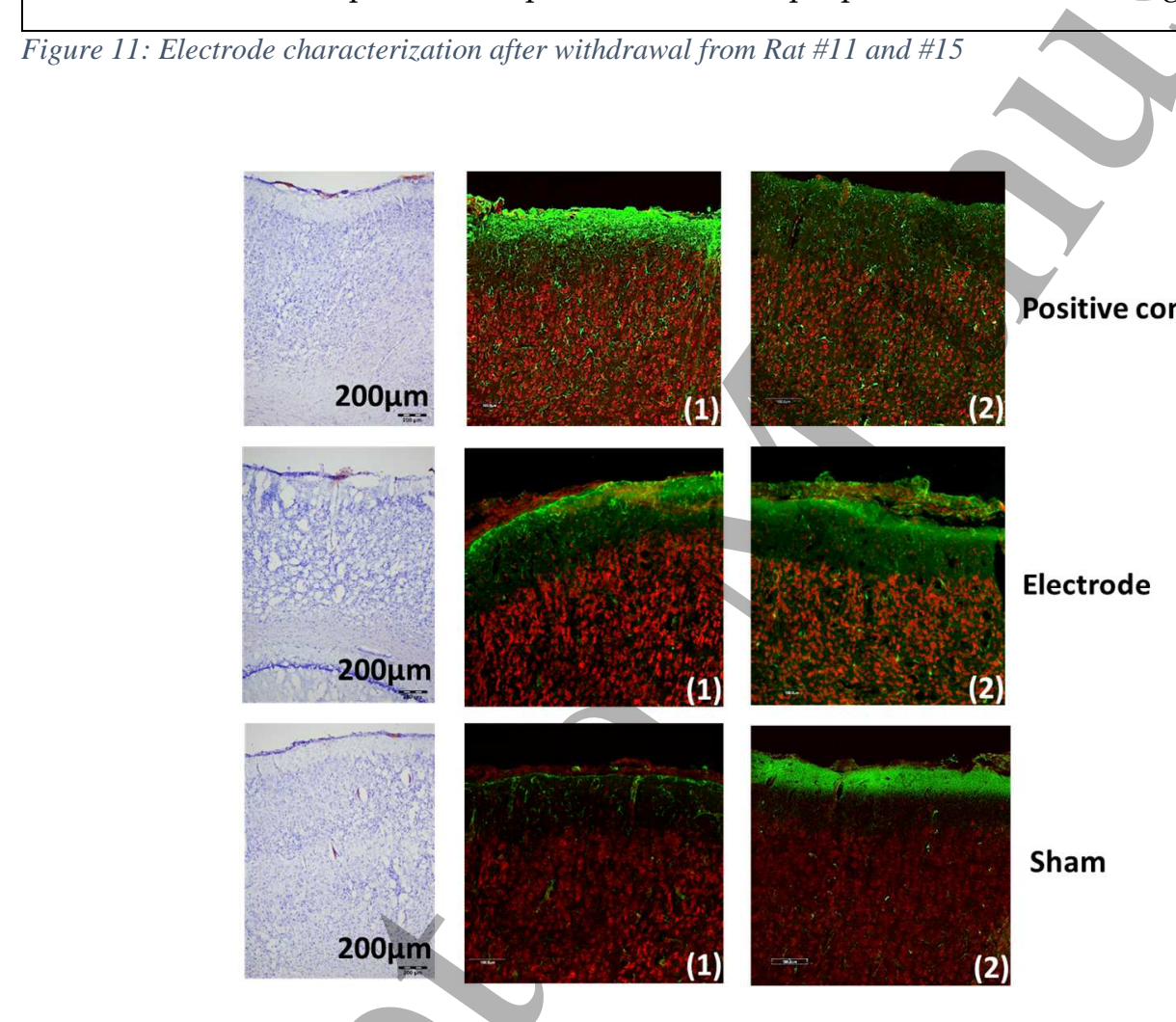

Figure 12: Left Nissl staining, Middle and right GFAP (1) and Ibal (2) immunostaining of the cortex below the electrode contact 


\begin{tabular}{|l|l|l|l|l|}
\hline MEA for rat \#9 & $\mathrm{R}_{1}(\mathrm{Ohm})$ & $\mathrm{Q}_{2}\left(\mathrm{~F} \cdot \mathrm{s}^{(\alpha-1)}\right)$ & $\alpha$ & $\mathrm{R}_{2}(\mathrm{Ohm})$ \\
\hline E1 & 480 & $0.515^{\mathrm{e}}-6$ & 0.801 & $0.721^{\mathrm{E}} 6$ \\
\hline E2 & 554 & $0.579^{\mathrm{e}}-6$ & 0.790 & $0.661^{\mathrm{E}} 6$ \\
\hline E3 & 458 & $0.334^{\mathrm{e}}-6$ & 0.802 & $0.726^{\mathrm{E}} 6$ \\
\hline E4 & 515 & $0.333^{\mathrm{e}}-6$ & 0.806 & $1.038^{\mathrm{E}} 6$ \\
\hline MEA for rat \#10 & $\mathrm{R}_{1}(\mathrm{Ohm})$ & $\mathrm{Q}_{2}\left(\mathrm{~F}^{(\alpha-1)}\right)$ & $\alpha$ & $\mathrm{R}_{2}(\mathrm{Ohm})$ \\
\hline E1 & 423 & $0.178^{\mathrm{e}}-6$ & 0.855 & $3.618^{\mathrm{E}} 6$ \\
\hline E2 & 470 & $0.160^{\mathrm{e}}-6$ & 0.856 & $4.733^{\mathrm{E}} 6$ \\
\hline E3 & 397 & $0.187^{\mathrm{e}}-6$ & 0.847 & $2.239^{\mathrm{E}} 6$ \\
\hline E4 & 461 & $0.183^{\mathrm{e}}-6$ & 0.862 & $1.900^{\mathrm{E}} 6$ \\
\hline
\end{tabular}

Table 1: Equivalent Model R1+R2/Q2 identification 